\title{
A Signature-Based Algorithm for Computing Gröbner Bases over Principal Ideal Domains
}

\author{
Maria Francis · Thibaut Verron
}

Received: 2 November 2018 / Revised: 3 April 2019 / Accepted: 16 April 2019 / Published online: 17 December 2019

(C) The Author(s) 2019

\begin{abstract}
Signature-based algorithms have become a standard approach for Gröbner basis computations for polynomial systems over fields, but how to extend these techniques to coefficients in general rings is not yet as well understood. In this paper, we present a proof-of-concept signature-based algorithm for computing Gröbner bases over commutative integral domains. It is adapted from a general version of Möller's algorithm (J Symb Comput 6(2-3), 345-359, 1988) which considers reductions by multiple polynomials at each step. This algorithm performs reductions with non-decreasing signatures, and in particular, signature drops do not occur. When the coefficients are from a principal ideal domain (e.g. the ring of integers or the ring of univariate polynomials over a field), we prove correctness and termination of the algorithm, and we show how to use signature properties to implement classic signature-based criteria to eliminate some redundant reductions. In particular, if the input is a regular sequence, the algorithm operates without any reduction to 0 . We have written a toy implementation of the algorithm in Magma. Early experimental results suggest that the algorithm might even be correct and terminate in a more general setting, for polynomials over a unique factorization domain (e.g. the ring of multivariate polynomials over a field or a PID).
\end{abstract}

Keywords Algorithms · Gröbner bases · Signature-based algorithms · Polynomials over rings · Principal ideal domains

\section{Mathematics Subject Classification 13P10}

\section{Introduction}

The theory of Gröbner bases was introduced by Buchberger in 1965 [5] and has since become a fundamental algorithmic tool in computer algebra. Over the past decades, many algorithms have been developed to compute

This work was started when the first author was supported by the Austrian FWF Grant Y464. The second author is supported by the Austrian FWF Grant F5004.

M. Francis

Department of Computer Science and Engineering, Indian Institute of Technology, Hyderabad, India

e-mail: mariaf@iith.ac.in

T. Verron $(\bowtie)$

Institute for Algebra, Johannes Kepler University, Linz, Austria

e-mail: thibaut.verron@jku.at 
Gröbner bases more and more efficiently. The latest iteration of such algorithms is the class of signature-based algorithms, which introduce the notion of signatures and use it to detect and prevent unnecessary or redundant reductions. Following early work in [20], the technique of signatures was first formally introduced for Algorithm F5 [11], allowing to compute a Gröbner basis for a regular sequence without any reduction to zero. Since then, there have been many research works in this direction $[2,7,8,13]$.

All these algorithms are for ideals in polynomial rings over fields. Gröbner bases can be defined and computed over commutative rings [1, Ch. 4]. This can be used in many applications, e.g. for polynomials over $\mathbb{Z}$ in lattice-based cryptography [12] or for polynomials over a polynomial ring as an elimination tool [21]. Many other examples are described in [18].

If the coefficient ring is not a field, there are two ways to define Gröbner bases, namely weak and strong bases. Strong Gröbner bases ensure that normal forms can be computed as in the case of fields. But a strong Gröbner basis is in general larger than a weak one, and if the base ring is not a Principal Ideal Domain (PID), then some ideals exist which do not admit a strong Gröbner basis. On the other hand, weak Gröbner bases, or simply Gröbner bases, always exist for polynomial ideals over a Noetherian commutative ring. They do not necessarily define a unique normal form, but they can be used to decide ideal membership. If necessary, over a PID, a post-processing phase performing coefficient reductions can be used to obtain a strong Gröbner basis.

Recent works have focused on generalizing signature-based techniques to Gröbner basis algorithms over rings. First steps in this direction, adding signatures to a modified version of Buchberger's algorithm for strong Gröbner bases over Euclidean rings [17], were presented in [9]. That paper proves that a signature-based Buchberger's algorithm for strong Gröbner bases cannot ensure correctness of the result after encountering a "signature-drop", but can nonetheless be used as a prereduction step in order to significantly speed up the computations.

In this paper, we prove that it is possible to compute a weak signature-Gröbner bases of polynomial ideals over PIDs (including Euclidean rings) using signature-based techniques. The proof-of-concept algorithm that we present is adapted from the weak Gröbner basis algorithm due to Möller [19] [1, Sec. 4.2], which is designed to compute a basis for a polynomial ideal over any ring, and does so by considering combinations and reductions by multiple polynomials at once. The main difference with the results of [9] is that we use a stricter definition of regular reductions, effectively preventing more reductions from happening, and at the same time adding more polynomials to the basis.

This ensures that no reductions leading to signature-drops can happen in the algorithm, and as a consequence, we prove that the algorithm terminates and computes a signature Gröbner basis with elements ordered with nondecreasing signatures. This property allows us to examine classic signature-based criteria, such as the syzygy criterion, the F5 criterion and the singular criterion, and show how they can be adapted to the case of PIDs. In particular, when the input forms a regular sequence, the algorithm performs no reductions to zero. To the best of our knowledge, this is the first algorithm that, given a regular sequence of polynomials with coefficients in a PID, can compute a Gröbner basis of the corresponding ideal without any reduction to zero.

Möller also presented an efficient algorithm that computes (strong) Gröbner basis for polynomial ideals where the coefficients are from Principal Ideal Rings [19, Sec. 4]. That algorithm skips the combinatorial bottleneck of computing saturated sets. Instead, it uses two polynomials to build S-polynomials and makes use of Gebauer-Möller criteria [15], previously introduced for fields, to discard redundant S-polynomials.

Whenever necessary, for clarity, we shall refer to that algorithm as Möller's strong algorithm. The algorithm at the center of our focus, computing weak Gröbner bases, will be referred to as Möller's weak algorithm, or simply Möller's algorithm.

We have written a toy implementation of the algorithms presented, with the F5 and the singular criteria, in the Magma Computational Algebra System [4], and compared its efficiency, in terms of number of excluded pairs, with Möller's strong algorithm. Experimentally, on all considered examples, Möller's (weak) algorithm with signatures does compute and reduce fewer S-polynomials than Möller's strong algorithm.

Möller's (weak) algorithm, without signatures, works for polynomial systems over any Noetherian commutative ring. The signature-based algorithm is only proved to be correct and to terminate for PIDs, but with very few changes, it can be made to accommodate inputs with coefficients in a more general ring. Interestingly, early experimental 
data with coefficients in a multivariate polynomial ring (a Unique Factorization Domain but not a PID) suggest that the signature-based algorithm might work over more general rings than just PIDs. For that reason, and because it does not over-complicate the exposition, we choose to present Möller's algorithms, with and without signatures, in their most general form, accepting input over any Noetherian commutative ring.

\subsection{Previous Works}

Signature-based Gröbner basis algorithms over fields have been extensively studied, and an excellent survey of those works can be found in [6]. The technical details of most proofs can be found in [10,22]. The theory of Gröbner bases for polynomials over Noetherian commutative rings dates back to the 1970s [19,23] and a good exposition of these approaches can be found in [1]. Algorithms exist for both flavors of Gröbner bases: Buchberger's algorithm [5] computes weak Gröbner bases over a PID, and Möller's weak algorithm [19] extends this approach to Noetherian commutative rings. As for strong Gröbner bases, they can be computed using an adapted version of Buchberger's algorithm [16] or Möller's strong algorithm [19]. Algorithms for computing signature Gröbner bases over Euclidean rings have been investigated in [9].

\section{Notations}

Let $R$ be a Noetherian integral domain, which is assumed to have a unit and be commutative. Let $A=R\left[x_{1}, \ldots, x_{n}\right]$ be the polynomial ring in $n$ indeterminates $x_{1}, \ldots, x_{n}$ over $R$. A monomial in $A$ is $x^{a}=x_{1}^{a_{1}} \ldots x_{n}^{a_{n}}$ where $a=\left(a_{1}, \ldots, a_{n}\right) \in \mathbb{N}^{n}$. A term is $k x^{a}$, where $k \in R$ and $k \neq 0$. We will denote all the terms in $A$ by $\operatorname{Ter}(A)$ and all the monomials in $A$ by $\operatorname{Mon}(A)$. We use the notation $\mathfrak{a}$ for polynomial ideals in $A=R\left[x_{1}, \ldots, x_{n}\right]$ and $I$ for ideals in the coefficient ring $R$.

The notion of monomial order can be directly extended from $\mathbb{K}\left[x_{1}, \ldots, x_{n}\right]$ to $A$. In the rest of the paper, we assume that $A$ is endowed with an implicit monomial order $\prec$, and we define as usual the leading monomial LM, the leading term LT and the leading coefficient LC of a given polynomial.

Given a tuple of polynomials $\left(g_{1}, \ldots, g_{s}\right)$ and $i \in\{1, \ldots, s\}$, we will frequently denote, for brevity, $M(i)=$ $\mathrm{LM}\left(g_{i}\right), C(i)=\mathrm{LC}\left(g_{i}\right)$ and $T(i)=\mathrm{LT}\left(g_{i}\right)=C(i) M(i)$.

\section{Gröbner Bases in Polynomial Rings over $R$}

For more details about the contents of this section, one can refer to [1, Chapter 4].

\subsection{Computations in $R$}

We assume that our coefficient ring $R$ is effective in the following sense.

(1) There are algorithms for arithmetic operations $(+, *$, zero test) in $R$.

(2) There is an algorithm LinDecomp:

- Input: $\left\{k_{1}, \ldots, k_{s}\right\} \subset R, k \in R$

- Output: TRUE iff $k \in\left\langle k_{1}, \ldots, k_{s}\right\rangle$ and if yes, $l_{1}, \ldots, l_{s} \in R$ such that $k=k_{1} l_{1}+\cdots+k_{s} l_{s}$.

(3) There is an algorithm Satldeal:

- Input: $\left\{k_{1}, \ldots, k_{s}\right\} \subset R, k \in R$

- Output: $\left\{l_{1}, \ldots, l_{r}\right\} \subset R$ generators of the saturated ideal $\left\langle k_{1}, \ldots, k_{s}\right\rangle:\langle k\rangle$. 
The condition that an algorithm LinDecomp exists is called linear equations being solvable in $R$ in [1, Def. 4.1.5].

Example Euclidean rings are effective, because one can implement those algorithms using GCD computations and Euclidean reductions. For example over $\mathbb{Z}, \operatorname{LinDecomp}(\{4\}, 12)$ is (TRUE, $\{3\})$, since 12 is in the ideal $\langle 4\rangle$ and $12=3 \cdot 4$. The output of Satldeal $(\{4\}, 6)$ is $\{2\}$ since $\langle 4\rangle:\langle 6\rangle=\frac{1}{6}(\langle 4\rangle \cap\langle 6\rangle)=\frac{1}{6}\langle 12\rangle=\langle 2\rangle$.

The ring of multivariate polynomials over a field is also effective, using Gröbner bases and normal forms to perform the same ideal computations.

\subsection{Weak Gröbner Bases over Rings}

For reduction in fields it is enough to check if the leading term of $f$ is divisible by the leading monomial of $g$ even though the actual reduction happens with the leading term of $g$. Clearly, in rings this is not a sufficient condition : $\mathrm{LC}(g)$ may not divide $\operatorname{LC}(f)$ even if $\operatorname{LM}(g)$ divides $\operatorname{LM}(f)$. Requiring that $\operatorname{LT}(g)$ divide $\operatorname{LT}(f)$ leads to the notion of strong Gröbner basis, more details can be found in [1, Sec. 4.5].

Here we are interested in computing weak Gröbner bases, and we recall the main definitions in this section. First, following $[1,19]$, we expand the definition of reduction to allow for a linear combination of reducers. We define saturated sets [1, Def.4.2.4] (called maximal sets in [19]).

Definition 3.1 Given a tuple of monomials $\left(x^{a_{1}}, \ldots, x^{a_{s}}\right)$, the saturated set for a monomial $x^{b}$ w.r.t. $\left(x^{a_{1}}, \ldots, x^{a_{s}}\right)$ is defined as

$\operatorname{Sat}\left(x^{b} ; x^{a_{1}}, \ldots, x^{a_{s}}\right)=\left\{i \in\{1, \ldots, s\}: x^{a_{i}} \mid x^{b}\right\}$.

A set $J \subseteq\{1, \ldots, s\}$ is said to be saturated w.r.t. $\left(x^{a_{1}}, \ldots, x^{a_{s}}\right)$ if $J=\operatorname{Sat}\left(M(J) ; x^{a_{1}}, \ldots, x^{a_{s}}\right)$ where $M(J)=$ $\operatorname{lcm}\left(x^{a_{i}}: i \in J\right)$. When clear from the context, we shall omit the list of monomials and write $J_{x^{b}}=\operatorname{Sat}\left(x^{b}\right)$.

Given a tuple of polynomials $\left(f_{1}, \ldots, f_{s}\right)$ and a set of indices $J \subset\{1, \ldots, s\}$, we denote by $I_{J}$ the ideal of $R$ defined as $I_{J}:=\left\langle\operatorname{LC}\left(f_{i}\right): i \in J\right\rangle$ and we define $M(J)=\operatorname{lcm}\left(\operatorname{LM}\left(f_{1}\right), \ldots, \operatorname{LM}\left(f_{s}\right)\right)$.

Definition 3.2 Let $f \in A$. Let $f_{1}, \ldots, f_{s} \in A$ and $x^{a_{1}}, \ldots, x^{a_{s}} \in \operatorname{Mon}(A)$ be such that $x^{a_{i}} \operatorname{LM}\left(f_{i}\right)=\operatorname{LM}(f)$ for all $i$. We say that we can weakly top reduce $f$ by $f_{1}, \ldots, f_{s} \in A$ if there exist $l_{1}, \ldots, l_{s}$ in $R$ such that

$\mathrm{LT}(f)=\sum_{i=1}^{s} l_{i} x^{a_{i}} \operatorname{LT}\left(f_{i}\right)$.

In our setting we will only perform top reductions, so we will simply call them weak reductions.

The outcome of the total reduction step is $g=f-\sum_{i=1}^{s} l_{i} x^{a_{i}} f_{i}$ and the $f_{i}$ 's are called the weak reducers. A polynomial $f \in A$ is weakly reducible if it can be weakly reduced, otherwise it is weakly reduced.

If $g$ is the outcome of reducing $f$, then $\operatorname{LM}(g) \prec \operatorname{LM}(f)$.

Example Consider the polynomial ring $\mathbb{Z}[x, y]$ with the lex ordering $y \prec x$, and consider the set $F=$ $\left\{f_{1}, f_{2}, f_{3}, f_{4}, f_{5}\right\}$ in $\mathbb{Z}[x, y]$, with $f_{1}=4 x y+x, f_{2}=3 x^{2}+y, f_{3}=5 x, f_{4}=4 y^{2}+y, f_{5}=5 y$. Let $f=2 x y+13 y-5$. We have $\mathrm{LT}(f)=2 x y=(2 y) \operatorname{LT}\left(f_{3}\right)-(2) \mathrm{LT}\left(f_{1}\right)$. This implies we can weakly reduce $f$ with $f_{1}, f_{3}$ to get $g=f-\left(2 y f_{3}-2 f_{1}\right)=2 x+13 y-5$.

We are now prepared to give the definition of (weak) Gröbner bases for an ideal in A.

Definition 3.3 Let $\mathfrak{a}$ be an ideal in $A$ and $G=\left\{g_{1}, \ldots, g_{t}\right\}$ be a finite set of nonzero polynomials in $\mathfrak{a}$. The set $G$ is called a weak Gröbner basis of $\mathfrak{a}$ in $A$ if it satisfies the following equivalent properties. 
1. $\langle\operatorname{LT}(G)\rangle=\langle\operatorname{LT}(\mathfrak{a})\rangle$;

2. for any $f \in \mathfrak{a}, f$ is weakly reducible modulo $G$;

3. for any $f \in A, f \in \mathfrak{a}$ if and only if $f$ weakly reduces to 0 modulo $G$.

Remark 3.4 Even though the notion of weak Gröbner bases is a weaker notion than that of strong Gröbner bases, one can use weak polynomial reductions to test for ideal membership. One can also define normal forms modulo a polynomial ideal. However, for those normal forms to be unique, one needs to perform further reductions on the coefficients, to "coset representative form", and one needs to perform reductions on non-leading coefficients as well [1, Th. 4.3.3]. Finally, note that, over a PID, one can easily recover a strong basis from a weak one [19, Th. 4].

\subsection{Möller's Algorithm for General Rings}

In this section, we present Möller's (weak) algorithm [19] for computing Gröbner bases over rings satisfying the conditions of Sect. 3.1. This algorithm is analogous to Buchberger's algorithm for rings, where the polynomial reduction is as defined above and S-polynomials are replaced with linear combinations of several (possibly more than 2) polynomials, defined in the following sense.

Consider a set $\left\{g_{1}, \ldots, g_{t}\right\}$ of polynomials. For $i \in\{1, \ldots, t\}$, let $M(i)=\operatorname{LM}\left(g_{i}\right), C(i)=\operatorname{LC}\left(g_{i}\right)$ and $T(i)=$ $\mathrm{LT}\left(g_{i}\right)$. Let $J$ be a saturated subset of $\{1, \ldots, t\}$ w.r.t. $\{M(1), \ldots, M(t)\}$. Recall that $M(J)=\operatorname{lcm}(M(j): j \in J)$. By definition, for all $j \in J, M(j)$ divides $M(J)$ and $J$ is maximal with this property.

Let $s \in J$ and $J^{*}=J \backslash\{s\}$. Similar to the idea behind S-polynomials, we want to eliminate the leading term $C(s) M(J)$ of $\frac{M(J)}{M(s)} g_{s}$. This can only be done if we multiply $\frac{M(J)}{M(s)} g_{s}$ by an element of the saturated ideal $\langle C(i): i \in J, i \neq s\rangle:\langle C(s)\rangle$. We want to consider all such multipliers, so we need to consider generators of this saturated ideal.

Let $c$ be such a generator, by definition $c C(s) \in\langle C(i): i \in J, i \neq s\rangle$ so there exists $\left(b_{i}\right)_{i \in J^{*}} \in R$ such that $c C(s)=\sum_{i \in J^{*}} b_{i} C(i)$. The (weak) S-polynomial associated with $J, s$ and $c$, for some suitable $\left(b_{i}\right)$, is defined as

$\mathrm{S}-\operatorname{Pol}\left(\left(g_{i}\right)_{i \in J^{*}} ; g_{s} ; c\right)=c \frac{M(J)}{M(s)} g_{s}-\sum_{i \in J^{*}} b_{i} \frac{M(J)}{M(i)} g_{i}$

If the ring $R$ is a PID, the saturated ideal $\langle C(i): i \in J, i \neq s\rangle:\langle C(s)\rangle$ admits a unique generator $c$ and we define

$C(J ; s)=\mathrm{LC}\left(c g_{s}\right)=c C(s)=\operatorname{lcm}\left(\operatorname{gcd}\left(\left\{C(j): j \in J^{*}\right\}\right), C(s)\right)$

$T(J ; s)=\mathrm{LT}\left(c g_{s}\right)=C(J ; s) M(J)$.

Then the S-polynomial associated with $J, s, c$, for some suitable $\left(b_{i}\right)$, can be written in the following form

$\mathrm{S}-\operatorname{Pol}\left(\left(g_{i}\right)_{i \in J^{*}} ; g_{s}\right)=\frac{T(J ; s)}{T(s)} g_{s}-\sum_{i \in J^{*}} b_{i} \frac{M(J)}{M(i)} g_{i}$.

Using this definition of S-polynomials, we recall Möller's algorithm (Algorithm 1) for computing a Gröbner basis of an ideal given by a set of generators over $R$. The correctness and termination of this algorithm are shown in [1, Th. 4.2.8 and Th. 4.2.9].

\section{Signatures in $A^{m}$}

We consider the free $A$-module $A^{m}$ with basis $\mathbf{e}_{1}, \ldots, \mathbf{e}_{m}$. A term (resp. monomial) in $A^{m}$ is $k x^{a} \mathbf{e}_{i}$ (resp. $x^{a} \mathbf{e}_{i}$ ) for some $k \in R \backslash\{0\}, x^{a} \in \operatorname{Mon}(A), i \in\{1, \ldots, m\}$. In this paper, terms in $A^{m}$ are ordered using the Position Over 

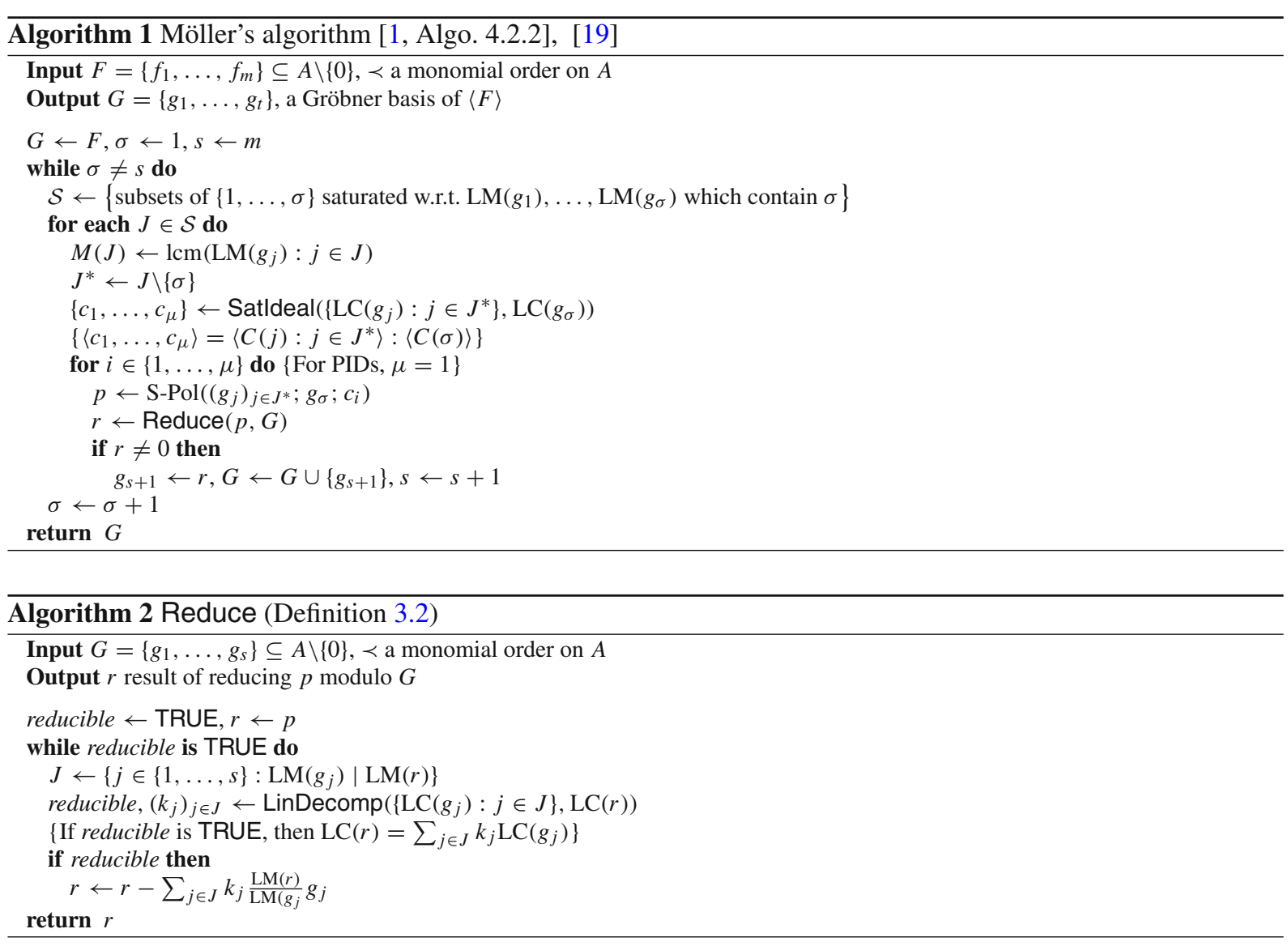

Term (POT) order, defined by

$k x^{a} \mathbf{e}_{i} \prec l x^{b} \mathbf{e}_{j} \Longleftrightarrow i \subsetneq j\left(\right.$ or $i=j$ and $\left.x^{a} \prec x^{b}\right)$.

Given two terms $k x^{a} \mathbf{e}_{i}$ and $l x^{b} \mathbf{e}_{j}$ in $A^{m}$, we write $k x^{a} \mathbf{e}_{i} \simeq l x^{b} \mathbf{e}_{j}$ if they are incomparable, i.e. if $a=b$ and $i=j$.

Given a set of polynomials $f_{1}, \ldots, f_{m} \in A$, elements of $A^{m}$ encode elements of the ideal $\left\langle f_{1}, \ldots, f_{m}\right\rangle$ through the $A$-module homomorphism ${ }^{-}: A^{m} \rightarrow A$, defined by setting $\overline{\mathbf{e}}_{i}=f_{i}$ and extending linearly to $A^{m}$. In particular, $\overline{\sum_{i=1}^{m} p_{i} \mathbf{e}_{i}}=\sum_{i=1}^{m} p_{i} f_{i}$.

We recall the concept of signatures in $A^{m}$. Let $\mathbf{p}=\sum_{i=1}^{m} p_{i} \mathbf{e}_{i}$ be a module element. Under the POT ordering, the signature of $\mathbf{p}$ is $\mathfrak{s}(\mathbf{p})=\mathrm{LT}\left(p_{i}\right) \mathbf{e}_{i}$ where $i$ is such that $p_{i+1}=\ldots=p_{m}=0$ and $p_{i} \neq 0$. Signatures are of the form $k x^{a} \mathbf{e}_{i}$, where $k \in R, x^{a} \in \operatorname{Mon}(A)$ and $\mathbf{e}_{i}$ is a standard basis vector.

Note that we have two ways of comparing two similar signatures $\mathfrak{s}(\boldsymbol{\alpha})=k x^{a} \mathbf{e}_{i}$ and $\mathfrak{s}(\boldsymbol{\beta})=l x^{b} \mathbf{e}_{j}$. We write $\mathfrak{s}(\boldsymbol{\alpha})=\mathfrak{s}(\boldsymbol{\beta})$ if $k=l, a=b$ and $i=j$, and we write $\mathfrak{s}(\boldsymbol{\alpha}) \simeq \mathfrak{s}(\boldsymbol{\beta})$ if $a=b$ and $i=j, k$ and $l$ being possibly different. If $R$ is a field, one can assume that the coefficient is 1 , and so this distinction is not important.

Note also that when we order signatures, we only compare the corresponding module monomials, and disregard the coefficients. This is a different approach from the one used in [9], where both signatures and coefficients are ordered.

Given a tuple $\left(\boldsymbol{\alpha}_{1}, \ldots, \boldsymbol{\alpha}_{s}\right)$ of module elements in $A^{m}$ and $i, j \in\{1, \ldots, s\}$, we shall frequently denote $S(i)=$ $\mathfrak{s}\left(\boldsymbol{\alpha}_{i}\right)$ for brevity.

In order to keep track of signatures we modify Definition 3.2 to introduce the notion of $\mathfrak{s}$-reduction. 
Definition 4.1 Let $\mathbf{p} \in A^{m}$. We say that we can signature-reduce (or $\mathfrak{s}$-reduce) $\mathbf{p}$ by $\boldsymbol{\beta}_{1}, \ldots, \boldsymbol{\beta}_{s} \in A^{m}$ if we can reduce $\overline{\mathbf{p}}$ by $\overline{\boldsymbol{\beta}}_{1}, \ldots, \overline{\boldsymbol{\beta}}_{s}$ (in the sense of Definition 3.2) and $\mathfrak{s}\left(x^{a_{i}} \boldsymbol{\beta}_{i}\right) \preceq \mathfrak{s}(\mathbf{p})$ for all $i=1, \ldots, s$, where $x^{a_{i}}=\frac{\operatorname{LM}(\overline{\mathbf{p}})}{\operatorname{LM}\left(\overline{\boldsymbol{\beta}}_{i}\right)}$. We can define similarly $\mathfrak{s}$-reduced module elements.

If $\mathfrak{s}\left(x^{a_{i}} \boldsymbol{\beta}_{i}\right) \simeq \mathfrak{s}(\mathbf{p})$ for some $i$ in the above $\mathfrak{s}$-reduction, then it is called a singular $\mathfrak{s}$-reduction step. Otherwise it is called a regular $\mathfrak{s}$-reduction step.

If $\mathfrak{s}\left(x^{a_{i}} \boldsymbol{\beta}_{i}\right) \simeq \mathfrak{s}(\mathbf{p})$ for exactly one $i$ and it is actually an equality $\mathfrak{s}\left(l_{i} x^{a_{i}} \boldsymbol{\beta}_{i}\right)=\mathfrak{s}(\mathbf{p})$, it is called a 1 -singular $\mathfrak{s - r e d u c t i o n ~ s t e p . ~}$

Remark 4.2 For simplicity, we only carry out weak top reductions, and in particular all s-reductions are weak top $\mathfrak{s}$-reductions. But performing regular $\mathfrak{s}$-reduction to eliminate trailing terms does not affect the correctness of the algorithm.

Just like $\mathfrak{s}$-reduction over fields, one can interpret $\mathfrak{s}$-reduction as polynomial reduction with an extra condition on the signature of the reducers. The difference with fields is that in $R\left[x_{1}, \ldots, x_{n}\right]$ polynomial reduction is defined differently from the classic polynomial reduction. Additionally, in the case of fields, all singular $\mathfrak{s}$-reductions are 1-singular.

The outcome $\mathbf{q}$ of $\mathfrak{s}$-reducing $\mathbf{p}$ is such that $\operatorname{LT}(\overline{\mathbf{q}}) \prec \operatorname{LT}(\overline{\mathbf{p}})$ and $\mathfrak{s}(\mathbf{q}) \preceq \mathfrak{s}(\mathbf{p})$. If $\mathbf{q}$ is the result of a regular $\mathfrak{s}$-reduction, then $\mathfrak{s}(\mathbf{q})=\mathfrak{s}(\mathbf{p})$. In signature-based algorithms, in order to keep track of the signatures of the basis elements, we only allow regular $\mathfrak{s}$-reductions. Later, we will also prove that elements which are 1-singular $\mathfrak{s}$-reducible can be discarded.

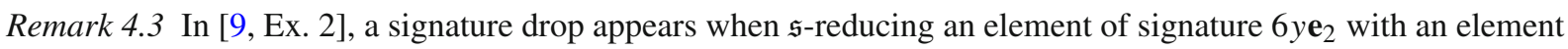
of signature $y \mathbf{e}_{2}$ causing the signature to "drop" to $5 y \mathbf{e}_{2}$. With our definition, since we only compare the module monomial part of the signatures, this is a (forbidden) singular $\mathfrak{s}$-reduction.

Definition 4.4 Let $\mathfrak{a}=\left\langle f_{1}, \ldots, f_{m}\right\rangle$ be an ideal in $A$. A finite subset $\mathcal{G}$ of $A^{m}$ is a (weak) signature Gröbner basis (or $\mathfrak{s}$-GB for short) of $\mathfrak{a}$ if all $\mathbf{u} \in A^{m} \mathfrak{s}$-reduce to zero $\bmod \mathcal{G}$.

Given a signature $\mathbf{T}$, we say that $\mathcal{G}$ is a (partial) signature Gröbner basis up to $\mathbf{T}$ if all $\mathbf{u} \in A^{m}$ with signature $\prec \mathbf{T} \mathfrak{s}$-reduce to $0 \bmod \mathcal{G}$.

Using this definition, we can give the following characterization of 1-singular reducibility, which allows for an easy algorithmic test.

Lemma 4.5 (Characterization of 1-singular $\mathfrak{s}$-reducibility) Let $\mathcal{G}=\left\{\boldsymbol{\alpha}_{1}, \ldots, \boldsymbol{\alpha}_{s}\right\} \subset A^{m}$ and $\mathbf{p} \in A^{m}$ such that $\mathcal{G}$ is a signature Gröbner basis up to signature $\mathfrak{s}(\mathbf{p})$. Then $\mathbf{p}$ is 1-singular $\mathfrak{s}$-reducible if and only if there exist $j \in\{1, \ldots, s\}$ and $k \in R$ and a monomial $x^{a}$ in A such that $\operatorname{LM}\left(x^{a} \overline{\boldsymbol{\alpha}}_{j}\right)=\operatorname{LM}(\overline{\mathbf{p}})$ and $k x^{a} \mathfrak{s}(\boldsymbol{\alpha} j)=\mathfrak{s}(\mathbf{p})$.

Proof If $\mathbf{p}$ is 1-singular $\mathfrak{s}$-reducible, then such $j, k$ and $x^{a}$ exist by definition. Conversely, given such $j, k$ and $x^{a}$, if $k x^{a} \operatorname{LT}\left(\overline{\boldsymbol{\alpha}}_{j}\right)=\mathrm{LT}(\overline{\mathbf{p}})$, then $\mathbf{p}$ is 1-singular $\mathfrak{s}$-reducible. If not, then $\operatorname{LM}\left(\overline{\mathbf{p}}-k x^{a} \overline{\boldsymbol{\alpha}}_{j}\right)=\operatorname{LM}(\overline{\mathbf{p}})$. Furthermore, $\mathfrak{s}\left(\mathbf{p}-k x^{a} \boldsymbol{\alpha}_{j}\right) \prec \mathfrak{s}(\mathbf{p})$, so $\mathbf{p}-k x^{a} \boldsymbol{\alpha}_{j} \mathfrak{s}$-reduces to 0 . In particular, there exist $\left(\mu_{i}\right)_{i \in\{1, \ldots, s\}}$ terms in $A$ such that for all $i$ with $\mu_{i} \neq 0, \operatorname{LM}\left(\mu_{i} \overline{\boldsymbol{\alpha}}_{i}\right)=\operatorname{LM}\left(\overline{\mathbf{p}}-k x^{a} \overline{\boldsymbol{\alpha}}_{j}\right), \operatorname{LT}\left(\overline{\mathbf{p}}-k x^{a} \overline{\boldsymbol{\alpha}}_{j}\right)=\sum_{i=1}^{s} \mu_{i} \operatorname{LT}\left(\overline{\boldsymbol{\alpha}}_{i}\right)$ and $\mu_{i} \mathfrak{s}\left(\boldsymbol{\alpha}_{i}\right) \preceq \mathfrak{s}\left(\mathbf{p}-k x^{a} \boldsymbol{\alpha}_{j}\right) \prec \mathfrak{s}(\mathbf{p})$. So putting together the two $\mathfrak{s}$-reductions, we obtain that

$\operatorname{LT}(\overline{\mathbf{p}})=k x^{a} \operatorname{LT}\left(\overline{\boldsymbol{\alpha}}_{j}\right)+\sum_{i=1}^{s} \mu_{i} \operatorname{LT}\left(\overline{\boldsymbol{\alpha}}_{i}\right)$

and this is a 1-singular $\mathfrak{s}$-reduction of $\mathbf{p}$.

We now define (weak) semi-strong signature Gröbner bases, which form a subclass of weak $\mathfrak{s}$-Gröbner bases. In the case of rings, it is easier to compute them than to directly compute weak $\mathfrak{s - G r o ̈ b n e r ~ b a s e s . ~}$ 
Definition 4.6 Let $\mathfrak{a}=\left\langle f_{1}, \ldots, f_{m}\right\rangle$ be an ideal in $A$. A finite subset $\mathcal{G}$ of $A^{m}$ is a semi-strong signature Gröbner basis (or s-s $\mathfrak{s}-\mathrm{GB}$ for short) of $\mathfrak{a}$ if, for all $\mathbf{u} \in A^{m}$,

- either $\mathbf{u}$ is (weakly) regular $\mathfrak{s}$-reducible modulo $\mathcal{G}$;

- or $\mathbf{u}$ is 1-singular $\mathfrak{s}$-reducible modulo $\mathcal{G}$;

- or $\overline{\mathbf{u}}=0$.

Given a signature $\mathbf{T}$, semi-strong signature Gröbner bases up to $\mathbf{T}$ are defined similarly by only considering module elements with signature $\prec \mathbf{T}$.

Lemma 4.7 ([6, Lem. 4.6]) Let $\mathfrak{a}=\left\langle f_{1}, \ldots, f_{m}\right\rangle$ be an ideal in $A$ and let $\mathcal{G} \subset A^{m}$. Then

1. If $\mathcal{G}$ is a $s-s \mathfrak{s}-G B$ of $\mathfrak{a}$, then $\mathcal{G}$ is $a \mathfrak{s}-G B$ of $\mathfrak{a}$.

2. If $\mathcal{G}$ is a $\mathfrak{s}-G B$ of $\mathfrak{a}$, then $\{\overline{\boldsymbol{\alpha}}: \boldsymbol{\alpha} \in \mathcal{G}\}$ is a Gröbner basis of $\mathfrak{a}$.

Proof The definition of a semi-strong Gröbner basis implies that all $\mathbf{u} \in A^{m}$ with $\overline{\mathbf{u}} \neq 0$ are s-reducible modulo $\mathcal{G}$, and so such $\mathfrak{s}$-reductions form a chain which can only terminate at 0 .

The proof that a signature Gröbner basis is a Gröbner basis is classical [6, Lem. 4.1].

In order to compute signature Gröbner bases, similar to the case of fields, we will restrict the computations to regular S-polynomials. For this purpose, we first introduce the signature of a set of indices, and regular sets.

Definition 4.8 Let $\mathcal{G}=\left(\boldsymbol{\alpha}_{1}, \ldots, \boldsymbol{\alpha}_{t}\right)$ be a tuple of module elements in $A^{m}$ and a set $J \subseteq\{1, \ldots, t\}$. For $i \in$ $\{1, \ldots, t\}$, let $M(i)=\operatorname{LM}\left(\overline{\boldsymbol{\alpha}}_{i}\right)$, and $S(i)=\mathfrak{s}\left(\boldsymbol{\alpha}_{i}\right)$. The presignature of $J$ is defined as

$S_{J}=\max _{s \in J}\left\{\frac{M(J)}{M(s)} S(s)\right\}$.

We say that $J$ is a regular set if there exists exactly one $s \in J$ such that $S_{J} \simeq \frac{M(J)}{M(s)} \mathfrak{s}\left(\boldsymbol{\alpha}_{s}\right)$. The index $s$ is called the signature index of $J$. We say that $J$ is a regular saturated set if $J \backslash\{s\}$ contains all $j$ such that $M(j) \mid M(J)$ and $\frac{M(J)}{M(j)} S(j) \prec S_{J}$.

Note that given a regular set $J$, one can always compute a regular saturated set $J^{\prime}$ containing $J$, by adding those indices $j$ such that $M(j) \mid M(J)$ and $\frac{M(J)}{M(j)} S(j) \prec S_{J}$.

Definition 4.9 Let $\left(\boldsymbol{\alpha}_{1}, \ldots, \boldsymbol{\alpha}_{t}\right)$ be a tuple of module elements in $A^{m}$. For $i \in\{1, \ldots, t\}$, let $M(i)=\operatorname{LM}\left(\overline{\boldsymbol{\alpha}}_{i}\right)$, $C(i)=\operatorname{LC}\left(\overline{\boldsymbol{\alpha}}_{i}\right)$ and $S(i)=\mathfrak{s}\left(\boldsymbol{\alpha}_{i}\right)$. Let $J \subset\{1, \ldots, t\}$ be a regular saturated set with signature index $s$, and let $J^{*}=J \backslash\{s\}$. Let $c$ be an element of a family of generators of $\left\langle C(j): j \in J^{*}\right\rangle:\langle C(s)\rangle$.

Let $\left(b_{j}\right)_{j \in J^{*}}$ be a tuple of elements of $R$ such that $c C(s)=\sum_{j \in J^{*}} b_{j} C(j)$. Then the (weak) S-polynomial associated with $J$ and $c$ is defined as

$\mathrm{S}-\operatorname{Pol}\left(\left(g_{j}\right)_{j \in J} ; c\right)=c \frac{M(J)}{M(s)} \boldsymbol{\alpha}_{s}-\sum_{j \in J^{*}} b_{j} \frac{M(J)}{M(j)} \boldsymbol{\alpha}_{j}$.

Its signature is

$S(J ; c)=\mathfrak{s}\left(\operatorname{S}-\operatorname{Pol}\left(\left(g_{j}\right)_{j \in J} ; c\right)\right)=c S_{J}=c \frac{M(J)}{M(s)} S(s)$.

Remark 4.10 When dealing with regular saturated sets, unlike in Sect. 3.2, we do not need to specify which $s \in J$ is singled out when computing the S-polynomial: the only possible $s$ is the signature index of $J$.

Remark 4.11 If the coefficient ring is a PID, the ideal $\left\langle C(j): j \in J^{*}\right\rangle:\langle C(s)\rangle$ is principal, and $c$ is uniquely determined up to an invertible factor. As such, it can be omitted, and in that case we shall simply write $S-\operatorname{Pol}(J)$ for the S-polynomial, and $S(J)$ for its signature. The signature can then be written as $S(J)=\frac{C(J)}{C(s)} S_{J}=\frac{C(J)}{C(s)} \frac{M(J)}{M(s)} S(s)$. 


\section{Adding Signatures to Möller's Weak Algorithm}

Recall that all $\mathfrak{s}$-reductions are weak top $\mathfrak{s}$-reductions. In this section, all S-polynomials are weak S-polynomials.

\subsection{Algorithms}

Algorithm SigMöller (Algorithm 3) is a signature-based version of Möller's algorithm which, given an ideal $\mathfrak{a}$ in $R\left[x_{1}, \ldots, x_{n}\right]$ where $R$ is a PID, computes a signature Gröbner basis of $\mathfrak{a}$.

The algorithm proceeds by maintaining a list of regular saturated sets $\mathcal{P}$ and computing weak S-polynomials obtained from these saturated sets. At each step, it selects the next regular saturated set $J \in \mathcal{P}$ such that $J$ has minimal presignature amongst elements of $\mathcal{P}$. This ensures that the algorithm computes new elements for the signature Gröbner basis with nondecreasing signatures (Prop. 5.2).

The algorithm then regular $\mathfrak{s}$-reduces these S-polynomials w.r.t. the previous elements, and adds to the basis those which are not equal to 0 and are not 1-singular $\mathfrak{5}$-reducible. Signature-based Gröbner basis algorithms over fields typically discard all new elements which are singular $\mathfrak{s}$-reducible, but this may be too restrictive for rings. On the other hand, the proof of Lemma 5.4 justifies that 1-singular $\mathfrak{s}$-reducible module elements can be safely discarded in the computations. The correctness of the criterion for 1-singular $\mathfrak{s}$-reducibility (Algorithm 4) was justified in Lemma 4.5. The correctness and termination of Algorithm SigMöller are proved in Th. 5.5 and Th. 5.6 respectively.

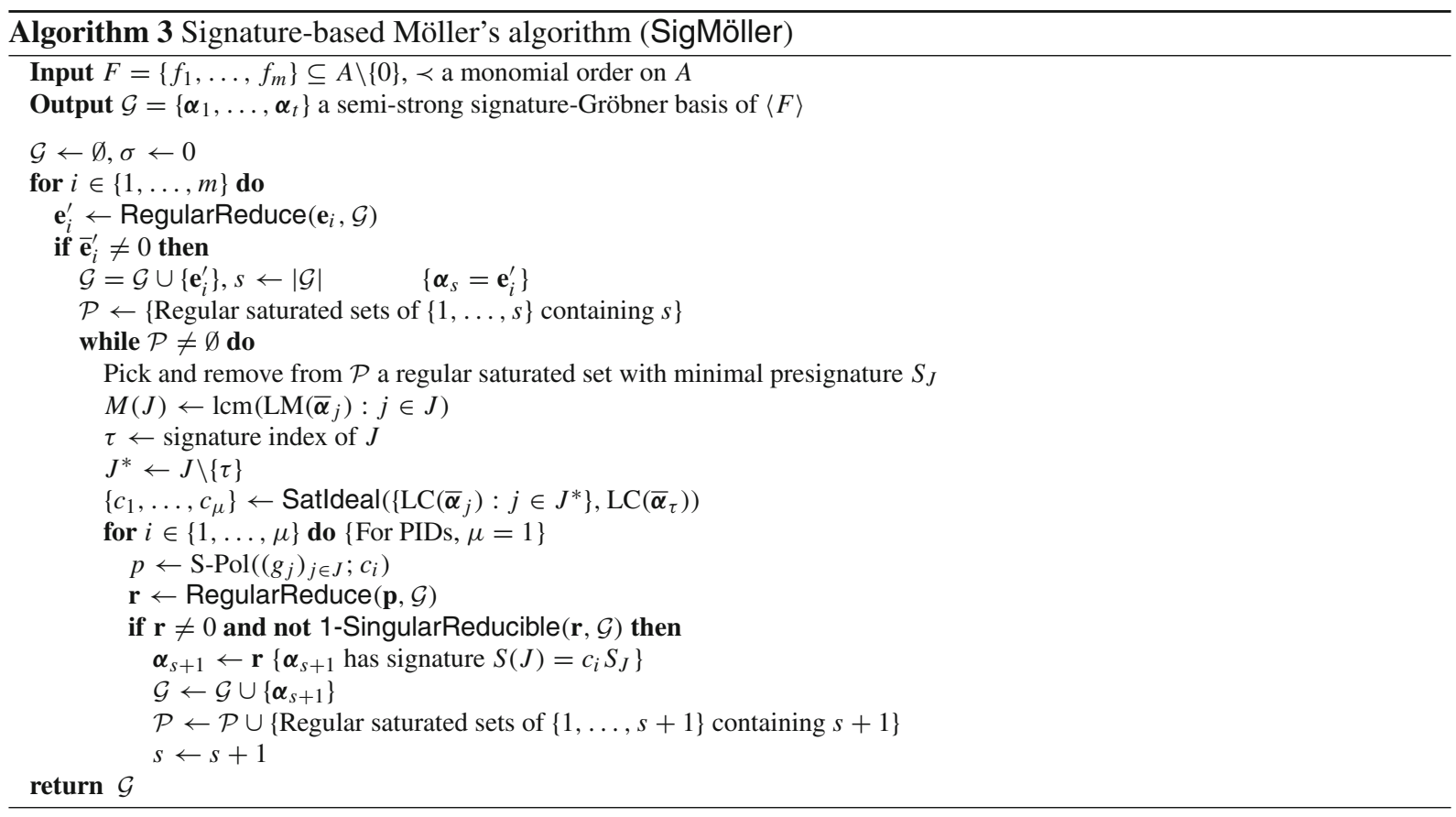

Due to space constraints, the subroutine RegularReduce is not explicitly written. It implements regular $\mathfrak{s}$ reduction of a module element $\mathbf{p}$ w.r.t. a set of module elements $\left\{\boldsymbol{\alpha}_{1}, \ldots, \boldsymbol{\alpha}_{s}\right\}$. It is a straightforward transposition of Reduce (Algorithm 2), with the additional condition that we only consider as reducers of $\mathbf{r}$ those $\boldsymbol{\alpha}_{j}$ with $\operatorname{LM}\left(\overline{\boldsymbol{\alpha}}_{j}\right) \mid \operatorname{LM}(\overline{\mathbf{r}})$ and $\frac{\operatorname{LM}(\overline{\mathbf{r}})}{\operatorname{LM}\left(\overline{\boldsymbol{\alpha}}_{j}\right)} \mathfrak{s}\left(\boldsymbol{\alpha}_{j}\right) \prec \mathfrak{s}(\mathbf{r})$.

Remark 5.1 Note that the algorithms, as presented, perform computations on module elements. However, for practical implementations, this represents a significant overhead. On the other hand, for any module element $\boldsymbol{\alpha}$, 
we only need its polynomial value $\bar{\alpha}$ and its signature $\mathfrak{s}(\boldsymbol{\alpha})$. Hence the algorithm only needs to keep track of the signatures of elements, which is made possible by the restriction to regular S-polynomials and regular $\mathfrak{s}$-reductions.

Example An example run of Algorithm 3 is available online. ${ }^{1}$

\subsection{Proof of Correctness}

In this section we prove the correctness of the algorithms presented in Sect. 5.1. The first result states that Algorithm SigMöller computes elements of the signature Gröbner basis in nondecreasing order on their signatures.

Proposition 5.2 Let $\left(\boldsymbol{\alpha}_{1}, \ldots, \boldsymbol{\alpha}_{t}\right)$ be the value of $\mathcal{G}$ at any point in the course of Algorithm SigMöller. Then $\mathfrak{s}\left(\boldsymbol{\alpha}_{1}\right) \preceq \mathfrak{s}\left(\boldsymbol{\alpha}_{2}\right) \preceq \cdots \preceq \mathfrak{s}\left(\boldsymbol{\alpha}_{t}\right)$.

Proof Assume that this is not the case, and let $i$ be the smallest index such that $\mathfrak{s}\left(\boldsymbol{\alpha}_{i}\right) \succ \mathfrak{s}\left(\boldsymbol{\alpha}_{i+1}\right)$. Let $J_{i}$ (resp. $\left.J_{i+1}\right)$ be the saturated set used to compute $\boldsymbol{\alpha}_{i}$ (resp. $\left.\boldsymbol{\alpha}_{i+1}\right)$. Note that $\mathfrak{s}\left(\boldsymbol{\alpha}_{i}\right) \simeq S\left(J_{i}\right)$ and $\mathfrak{s}\left(\boldsymbol{\alpha}_{i+1}\right) \simeq S\left(J_{i+1}\right)$.

If $i \notin J_{i+1}$, then $J_{i+1}$ was already in the queue $\mathcal{P}$ when $J_{i}$ was selected, and so, by the selection criterion in the algorithm, $S\left(J_{i}\right) \preceq S\left(J_{i+1}\right)$.

If $i \in J_{i+1}$, then $S\left(J_{i+1}\right) \succeq \frac{x^{J_{i+1}}}{\operatorname{LM}\left(\overline{\boldsymbol{\alpha}}_{i}\right)} \mathfrak{s}\left(\boldsymbol{\alpha}_{i}\right) \succeq \mathfrak{s}\left(\boldsymbol{\alpha}_{i}\right)$.

The following useful lemma gives consequences of the fact that two regular $\mathfrak{s}$-reduced elements share the same signature.

Lemma 5.3 Let $\mathcal{G}=\left(\boldsymbol{\alpha}_{1}, \ldots, \boldsymbol{\alpha}_{s}\right)$ be a signature Gröbner basis up to signature $\mathbf{L}$.

Let $\mathbf{p}, \mathbf{q} \in A^{m}$ such that $\mathfrak{s}(\mathbf{p})=\mathfrak{s}(\mathbf{q})=\mathbf{L}$, and $\mathbf{p}$ and $\mathbf{q}$ are regular $\mathfrak{s}$-reduced. Then $\mathrm{LM}(\overline{\mathbf{p}})=\mathrm{LM}(\overline{\mathbf{q}})$ and either $\operatorname{LT}(\overline{\mathbf{p}})=\operatorname{LT}(\overline{\mathbf{q}})$, or $\operatorname{LC}(\overline{\mathbf{p}}-\overline{\mathbf{q}})$ lies in the ideal

$C:=\left\langle\operatorname{LC}\left(\overline{\boldsymbol{\alpha}_{j}}\right): \operatorname{LM}\left(\overline{\boldsymbol{\alpha}_{j}}\right)\right| m$ and $\left.\frac{m}{\operatorname{LM}\left(\overline{\boldsymbol{\alpha}_{j}}\right)} \mathfrak{s}\left(\boldsymbol{\alpha}_{j}\right) \not \mathfrak{s}(\mathbf{p})\right\rangle$.

Proof Let $\mathbf{r}=\mathbf{p}-\mathbf{q}$. Since $\mathfrak{s}(\mathbf{p})=\mathfrak{s}(\mathbf{q})$, we have $\mathfrak{s}(\mathbf{r}) \prec \mathfrak{s}(\mathbf{p})=\mathbf{L}$, and so $\mathbf{r} \mathfrak{s}$-reduces to 0 modulo $\mathcal{G}$. Assume first that $\operatorname{LM}(\overline{\mathbf{p}}) \neq \operatorname{LM}(\overline{\mathbf{q}})$, then w.l.o.g. we may assume that $\operatorname{LM}(\overline{\mathbf{p}}) \succ \operatorname{LM}(\overline{\mathbf{q}})$, so $\operatorname{LM}(\overline{\mathbf{r}})=\operatorname{LM}(\overline{\mathbf{p}})$. Since $\mathbf{r}$ is regular $\mathfrak{s}$-reducible, $\mathbf{p}$ is $\mathfrak{s}$-reducible. This is a contradiction with the assumption that $\mathbf{p}$ is $\mathfrak{s}$-reduced.

So $\operatorname{LM}(\overline{\mathbf{p}})=\operatorname{LM}(\overline{\mathbf{q}})=: m$. If $\operatorname{LT}(\overline{\mathbf{p}}) \neq \operatorname{LT}(\overline{\mathbf{q}}), C$ is the ideal of leading coefficients of polynomials which can eliminate $m$, and since $\mathbf{r}$ is $\mathfrak{s}$-reducible, $\operatorname{LC}(\overline{\mathbf{p}})-\operatorname{LC}(\overline{\mathbf{q}}) \in C$.

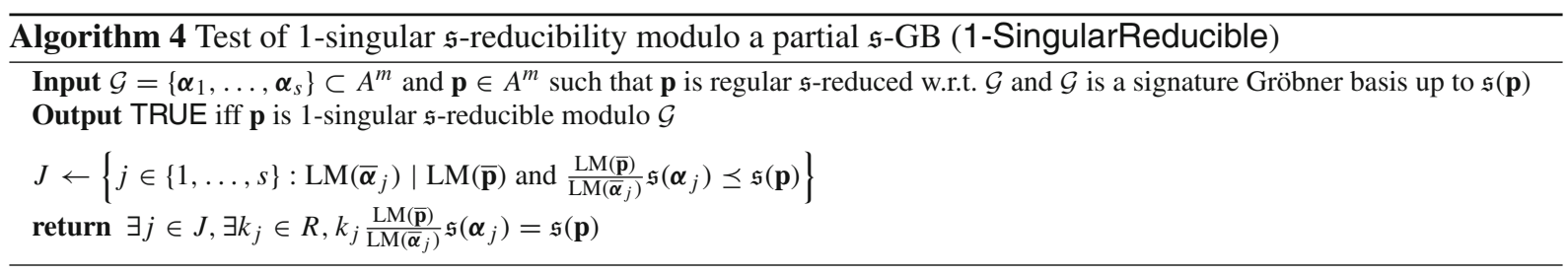

We now prove the correctness of Algorithm SigMöller. The proof follows the structure of the proof in the case of fields [22], and adapts it to Möller's algorithm over PIDs. In particular, it takes into account weak $\mathfrak{s - r e d u c t i o n s}$ instead of classical $\mathfrak{s}$-reductions. The algorithm ensures that all regular S-polynomials up to a given signature $\mathbf{T}$ $\mathfrak{s}$-reduce to 0 , and proving the correctness of the algorithm requires proving that this implies that all module elements with signature $\prec \mathbf{T} \mathfrak{s}$-reduce to 0 .

The key lemma of the proof is the following.

$\overline{1}$ https://github.com/ThibautVerron/SignatureMoller/blob/master/ACA18_example.pdf. 
Lemma 5.4 Let $\mathcal{G}=\left(\boldsymbol{\alpha}_{1}, \ldots, \boldsymbol{\alpha}_{s}\right) \subseteq A^{m}$. Let $\mathbf{u} \in A^{m} \backslash\{0\}$ be $\mathfrak{s}$-reduced such that $\overline{\mathbf{u}} \neq 0$. Assume that $\mathcal{G}$ is a s-s $\mathfrak{s}-G B$ basis up to signature $\mathfrak{s}(\mathbf{u})$. Then there exists an $S$-polynomial $\mathbf{p}$ w.r.t. $\mathcal{G}$, such that:

1. the signature of $\mathbf{p}$ divides the signature of $\mathbf{u}: k x^{a} \mathfrak{s}(\mathbf{p})=\mathfrak{s}(\mathbf{u})$ with $k \in R$ and $x^{a} \in \operatorname{Mon}(A)$;

2. if $\mathbf{p}^{\prime}$ is the result of regular $\mathfrak{s}$-reducing $\mathbf{p}$ w.r.t. $\mathcal{G}$, then $k x^{a} \mathbf{p}^{\prime}$ is regular $\mathfrak{s}$-reduced.

Proof The proof is in two steps: first, we construct a S-polynomial $\mathbf{p}$ whose signature divides $\mathfrak{s}(\mathbf{u})$, and then, starting from $\mathbf{p}$, we show that there exists an S-polynomial satisfying the conditions of the lemma.

In the remainder of the proof, for $i \in\{1, \ldots, s\}$, let $M(i)=\operatorname{LM}\left(\overline{\boldsymbol{\alpha}}_{i}\right), C(i)=\operatorname{LC}\left(\overline{\boldsymbol{\alpha}}_{i}\right), T(i)=\operatorname{LT}\left(\overline{\boldsymbol{\alpha}}_{i}\right)$ and $S(i)=\mathfrak{s}\left(\boldsymbol{\alpha}_{i}\right)$.

Existence of a S-polynomial satisfying 1 For the first step, let $\mathfrak{s}(\mathbf{u})$ be $l x^{b} \mathbf{e}_{i}$ for some $l \in R, x^{b}$ a monomial and $\mathbf{e}_{i}$ a basis vector. Let $\mathbf{e}_{i}^{\prime}$ be the result of regular $\mathfrak{s}$-reducing $\mathbf{e}_{i}$. If $\overline{\mathbf{e}}_{i}^{\prime}=0$, then $\mathbf{u}$ regular $\mathfrak{s}$-reduces to 0 , which is a contradiction since we assumed $\mathbf{u}$ to be $\mathfrak{s}$-reduced and $\overline{\mathbf{u}} \neq 0$. Let $\mathbf{L}=l x^{b} \mathbf{e}_{i}^{\prime}$, it has signature $l x^{b} \mathbf{e}_{i}$. Then $\mathbf{u}-\mathbf{L}$ has a smaller signature than $\mathbf{u}$, so it $\mathfrak{s}$-reduces to zero and in particular it is $\mathfrak{s}$-reducible. Also, $\mathbf{L}$ is $\mathfrak{s}$-reducible by $\mathbf{e}_{i}^{\prime}$. Consider the sum $(\mathbf{u}-\mathbf{L})+\mathbf{L}=\mathbf{u}$. It is not $\mathfrak{s}$-reducible, which implies that $\mathrm{LT}(\overline{\mathbf{u}-\mathbf{L}})=-\mathbf{L T}(\overline{\mathbf{L}})$.

Let $J_{\mathrm{LM}(\overline{\mathbf{L}})}$ be the maximal regular saturated set $J$ with $M(J) \mid \operatorname{LM}(\overline{\mathbf{L}})$. Since $\mathbf{u}-\mathbf{L} \mathfrak{s}$-reduces to zero, there exists $\left(m_{j}\right)_{j \in J_{\mathrm{LM}(\overline{\mathbf{L}})}}$ monomials in $A$, and $\left(k_{j}\right)_{j \in J_{\mathrm{LM}(\overline{\mathbf{L}})}}$ coefficients in $R$ such that

$\mathrm{LT}(\overline{\mathbf{u}}-\overline{\mathbf{L}})=\sum_{j \in J_{\mathrm{LM}(\overline{\mathbf{L}})}} k_{j} m_{j} T(j)$

with $m_{j} M(j)=\operatorname{LM}(\overline{\mathbf{u}}-\overline{\mathbf{L}})$ and $\mathfrak{s}\left(k_{j} m_{j} \boldsymbol{\alpha}_{j}\right)=k_{j} m_{j} S(j) \preceq \mathfrak{s}(\mathbf{u}-\mathbf{L}) \prec \mathfrak{s}(\mathbf{u})$ for all $i$ such that $k_{j} \neq 0$. Let $\sigma$ be the index of $\mathbf{e}_{i}^{\prime}$ in $\mathcal{G}$, that is $\boldsymbol{\alpha}_{\sigma}=\mathbf{e}_{i}^{\prime}$. Consider the set $J^{\prime}=\left\{j: m_{j} \neq 0\right\} \cup\{\sigma\} \subseteq J_{\mathrm{LM}(\overline{\mathbf{L}})}$, it is regular by construction.

Let $J$ be a regular saturated set containing $J^{\prime}$. Then, since for all $j \in J^{\prime}, M(j) \mid \operatorname{LM}(\overline{\mathbf{L}})=x^{b} M(\sigma), M(J)=$ $\operatorname{lcm}\left\{M(j): j \in J^{\prime}\right\} \mid x^{b} M(\sigma)$. Furthermore, looking at the leading coefficients in Eq. (5.1), we have

$l C(\sigma)=-\sum_{j \in J^{\prime}} k_{j} C(j)$

and so $l \in\langle C(j): j \in J, j \neq \sigma\rangle:\langle C(\sigma)\rangle$. Since $R$ is a PID, this ideal is principal. Let $b_{J}$ be its generator, then $b_{J} \mid l$. Let $\mathbf{p}$ be the S-polynomial corresponding to $J$ and $b_{J}$. It is regular by construction since $J$ is a regular saturated set, and its signature is $\mathfrak{s}(\mathbf{p})=b_{J} \frac{M(J)}{M(\sigma)} S(\sigma)=b_{J} \frac{M(J)}{\operatorname{LM}\left(\overline{\mathbf{e}}_{i}^{\prime}\right)} \mathbf{e}_{i}$. Since $b_{J}$ divides $l$ and $M(J)$ divides $x^{b} M(\sigma)$, $\mathfrak{s}(\mathbf{p})$ divides $l x^{b} s \mathbf{e}_{i}^{\prime}=\mathfrak{s}(\mathbf{L})=\mathfrak{s}(\mathbf{u})$.

Existence of a S-polynomial satisfying 1 . and 2 Let $\mathbf{p}$ be an S-polynomial whose signature divides $\mathfrak{s}(\mathbf{u})$, and let $\mathbf{p}^{\prime}$ be the regular $\mathfrak{s}$-reduced form of $\mathbf{p}$. Write $\mathfrak{s}(\mathbf{u})=\mathfrak{s}\left(k x^{a} \mathbf{p}\right)$, where $k \in R$ and $x^{a}$ is a monomial.

We can assume that $k x^{a} \mathbf{p}^{\prime}$ is regular $\mathfrak{s}$-reducible or else we are done. We then construct an S-polynomial $\mathbf{q}$ such that $\mathfrak{s}\left(l x^{b} \mathbf{q}\right)=\mathfrak{s}(\mathbf{u})$ and $\operatorname{LM}\left(\overline{k x^{a} \mathbf{p}}\right) \succ \operatorname{LM}\left(\overline{l x^{b} \mathbf{q}}\right)$. If $l x^{b} \mathbf{q}^{\prime}$, where $\mathbf{q}^{\prime}$ is obtained by regular $\mathfrak{s}$-reducing $\mathbf{q}$, is not regular $\mathfrak{s}$-reducible then we are done. Otherwise we can do the same process again and get a third S-polynomial with the same properties and keep repeating. Since the initial terms are strictly decreasing and we have a well order there are only finitely many such S-polynomials.

First, we show that we can assume that $x^{a} \succ 1$. Indeed, assume that $a=0$ and $k \mathbf{p}^{\prime}$ is regular $\mathfrak{s}$-reducible. Since $R$ is an integral domain, $\operatorname{LM}\left(k \overline{\mathbf{p}}^{\prime}\right)=\operatorname{LM}\left(\overline{\mathbf{p}}^{\prime}\right)$. Let $J_{\mathrm{LM}\left(\overline{\mathbf{p}}^{\prime}\right)}$ be the maximal regular saturated set $J$ with $M(J) \mid \operatorname{LM}\left(\overline{\mathbf{p}}^{\prime}\right)$.

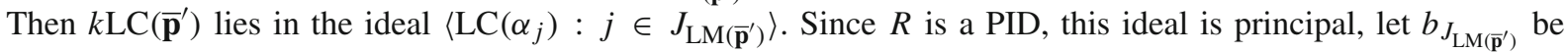
its generator, then $b_{J_{\mathrm{LM}\left(\overline{\mathbf{p}}^{\prime}\right)}} \mid k$. Let $\overline{\mathbf{q}}$ be the S-polynomial corresponding to the regular saturated set $J_{\mathrm{LM}\left(\overline{\mathbf{p}}^{\prime}\right)}$ and the generator $b_{J_{\mathrm{LM}\left(\overline{\mathbf{p}}^{\prime}\right)}}$, its signature divides $\mathfrak{s}(\mathbf{u})$ and is strictly divisible by $\mathfrak{s}(\mathbf{p})$. Repeating the process as needed, we obtain a strictly increasing sequence of elements dividing the coefficient of $\mathfrak{s}(\mathbf{u})$, and since $R$ is a PID and in particular a unique-factorization domain, this sequence has to be finite. So we can assume that $x^{a} \succ 1$. 
We will construct two reductions of $\operatorname{LT}\left(k x^{a} \overline{\mathbf{p}}^{\prime}\right)$, which taken together will give the S-polynomial $\mathbf{q}$. For the first reduction, the module element $\mathbf{p}^{\prime} \in A^{m}$ is regular $\mathfrak{s}$-reduced modulo the s-s $\mathfrak{s}$-GB $\mathcal{G}$, and its signature is smaller than $\mathfrak{s}(\mathbf{u})$. Furthermore, by assumption $k x^{a} \mathbf{p}^{\prime}$ is not regular $\mathfrak{s}$-reduced, so $\overline{\mathbf{p}}^{\prime}$ cannot be 0 . So, by definition of a s-s $\mathfrak{s}$-GB, $\mathbf{p}^{\prime}$ is 1 -singular $\mathfrak{s}$-reducible. So there exists $\left(t_{i}^{(1)}\right)_{i \in J_{1}}$ terms in $A$, with $J_{1} \subset\{1, \ldots, s\}$ and for all $i \in J_{1}$, $t_{i}^{(1)} \neq 0$, and such that

$\mathrm{LT}\left(\overline{\mathbf{p}}^{\prime}\right)=\sum_{i \in J_{1}} t_{i}^{(1)} \mathrm{LT}\left(\overline{\boldsymbol{\alpha}}_{i}\right)=\sum_{i \in J_{1}} t_{i}^{(1)} T(i)$

with for all $i \in J_{1}, \operatorname{LM}\left(t_{i}^{(1)} \overline{\boldsymbol{\alpha}}_{i}\right)=\operatorname{LM}\left(t_{i}^{(1)}\right) M(i)=\operatorname{LM}\left(\overline{\mathbf{p}}^{\prime}\right)$. Furthermore, there exists $\tau$ in $J_{1}, t_{\tau}^{(1)} S(\tau)=\mathfrak{s}(\overline{\mathbf{p}})$ and for all $i \in J_{1} \backslash\{\tau\}, t_{i}^{(1)} S(i) \prec \mathfrak{s}(\mathbf{p})$.

We now build the second reduction. Since $k x^{a} \mathbf{p}^{\prime}$ is regular $\mathfrak{s}$-reducible, there exists $\left(t_{i}^{(2)}\right)_{i \in J_{2}}$ terms in $A$, with $J_{2} \subset\{1, \ldots, s\}$ and for all $i \in J_{2}, t_{i}^{(2)} \neq 0$, such that

$\mathrm{LT}\left(k x^{a} \overline{\mathbf{p}}^{\prime}\right)=\sum_{i \in J_{2}} t_{i}^{(2)} \mathrm{LT}\left(\overline{\boldsymbol{\alpha}}_{i}\right)=\sum_{i \in J_{2}} t_{i}^{(2)} T(i)$,

and for all $j \in J_{2}, \operatorname{LM}\left(t_{j}^{(2)}\right) M(j)=\operatorname{LM}\left(k x^{a} \overline{\mathbf{p}}^{\prime}\right)$ and $t_{j}^{(2)} S(j) \prec \mathfrak{s}\left(k x^{a} \mathbf{p}^{\prime}\right)$.

Now let $J=J_{1} \cup J_{2}$, and let $t_{i}^{(1)}=0$ if $i \in J_{2} \backslash J_{1}, t_{j}^{(2)}=0$ if $j \in J_{1} \backslash J_{2}$. Note that $\tau \notin J_{2}$, so $t_{\tau}^{(2)}=0$. Combining Eqs. (5.2) and (5.3), we obtain a decomposition of $k x^{a} t_{\tau} T(\tau)$ as

$k x^{a} t_{\tau} T(\tau)=-\sum_{i \in J \backslash\{\tau\}} t_{i} T(i)$

where for all $i \in J, t_{i}=k x^{a} t_{i}^{(1)}-t_{i}^{(2)}$. Furthermore, for all $i \in J \backslash\{\tau\}, \operatorname{LM}\left(t_{i}\right) M(i)=\operatorname{LM}\left(x^{a} \overline{\mathbf{p}}^{\prime}\right)=\operatorname{LM}\left(x^{a} t_{\tau}\right) M(\tau)$ and $t_{i} S(i) \prec \mathfrak{s}(\overline{\mathbf{p}})=k x^{a} t_{\tau} S(\tau)$.

The same argument as the one used, in the first part of the proof, to construct an S-polynomial based on Eq. (5.1) yields an S-polynomial $\mathbf{q}$ such that $\mathfrak{s}(\mathbf{q})$ divides $\mathfrak{s}(\mathbf{u})$, say $l x^{b} \mathfrak{s}(\mathbf{q})=\mathfrak{s}(\mathbf{u})$. Furthermore, since the leading term is eliminated in the construction of an S-polynomial, LT $\left(l x^{b} \overline{\mathbf{q}}\right) \prec \mathrm{LT}\left(k x^{a} \overline{\mathbf{p}}^{\prime}\right)$, which concludes the proof.

Theorem 5.5 (Correctness of Algorithm SigMöller) Let $\mathbf{T}$ be a term of $A^{m}$ and let $\mathcal{G}=\left(\boldsymbol{\alpha}_{1}, \ldots, \boldsymbol{\alpha}_{s}\right) \subseteq A^{m}$ be a finite basis as computed by Algorithm 3. Assume that all regular S-polynomials $\mathbf{p}$ with $\mathfrak{s}(\mathbf{p}) \prec \mathbf{T} \mathfrak{s}$-reduce to 0 w.r.t. $\mathcal{G}$. Then $\mathcal{G}$ is a semi-strong signature-Gröbner basis up to signature $\mathbf{T}$.

Proof To get a contradiction assume there exists a $\mathbf{u} \in A^{m}$ with $\mathfrak{s}(\mathbf{u}) \prec \mathbf{T}$ such that $\mathbf{u}$ does not $\mathfrak{s}$-reduce to zero. Assume w.l.o.g. that $\mathfrak{s}(\mathbf{u})$ is $\prec$-minimal such that $\mathbf{u}$ does not $\mathfrak{s}$-reduce to zero and also that $\mathbf{u}$ is regular $\mathfrak{s}$-reduced.

By Lemma 5.4 there is an S-polynomial $\mathbf{p}$ with $\mathfrak{s}\left(k x^{a} \mathbf{p}\right)=\mathfrak{s}(\mathbf{u})$ with $k \in R, x^{a} \in \operatorname{Mon}(A)$. Also, $k x^{a} \mathbf{p}^{\prime}$ is regular $\mathfrak{s}$-reduced where $\mathbf{p}^{\prime}$ is the result of regular $\mathfrak{s}$-reducing $\mathbf{p}$.

Let $J_{\mathrm{LM}(\overline{\mathbf{u}})}$ be the maximal regular saturated set $J$ with $M(J) \mid \operatorname{LM}(\overline{\mathbf{u}})$. Since $\mathfrak{s}\left(k x^{a} \mathbf{p}\right)=\mathfrak{s}(\mathbf{u})$ and both $k x^{a} \mathbf{p}^{\prime}$ and $\mathbf{u}$ are regular $\mathfrak{s}$-reduced, we have by Lemma 5.3 that $\operatorname{LM}\left(k x^{a} \overline{\mathbf{p}}^{\prime}\right)=\operatorname{LM}(\overline{\mathbf{u}})$, and either $\operatorname{LT}\left(k x^{a} \overline{\mathbf{p}}^{\prime}\right)=\operatorname{LT}(\overline{\mathbf{u}})$, or

$\operatorname{LC}\left(\overline{\mathbf{u}}-k x^{a} \overline{\mathbf{p}}^{\prime}\right) \in\left\langle\operatorname{LC}\left(\overline{\boldsymbol{\alpha}}_{j}\right): j \in J_{\mathrm{LM}(\overline{\mathbf{u}})}\right\rangle$.

So in either case, there exists $\left(t_{i}\right)_{i \in J_{\mathrm{LM}(\overline{\mathrm{u}})}}$ terms in $A$, possibly all zero, such that

$\mathrm{LT}(\overline{\mathbf{u}})-\mathrm{LT}\left(k x^{a} \overline{\mathbf{p}}^{\prime}\right)=\sum_{i \in J_{\mathrm{LM}(\overline{\mathbf{u}})}} t_{i} \mathrm{LT}\left(\overline{\boldsymbol{\alpha}}_{i}\right)$ 
and $t_{i} \operatorname{LM}\left(\overline{\boldsymbol{\alpha}}_{i}\right)=\operatorname{LM}(\overline{\mathbf{r}})=\operatorname{LM}(\overline{\mathbf{u}})$ for all $i$ such that $t_{i} \neq 0$.

Since $\mathbf{p}^{\prime}$ is a regular S-polynomial with $\mathfrak{s}\left(\mathbf{p}^{\prime}\right) \preceq \mathfrak{s}(\mathbf{u}) \prec \mathbf{T}, \mathbf{p}^{\prime}$ is $\mathfrak{s}$-reducible, and so $k x^{a} \mathbf{p}^{\prime}$ is $\mathfrak{s}$-reducible. So there exists $\left(\tau_{i}\right)_{i \in J_{\mathrm{LM}(\overline{\mathbf{u}})}}$ terms in $A$ such that

$\mathrm{LT}\left(k x^{a} \overline{\mathbf{p}}^{\prime}\right)=\sum_{i \in J_{\mathrm{LM}(\overline{\mathbf{u}})}} \tau_{i} \mathrm{LT}\left(\overline{\boldsymbol{\alpha}}_{i}\right)$,

and $\tau_{i} \operatorname{LM}\left(\overline{\boldsymbol{\alpha}}_{i}\right)=\operatorname{LM}\left(k x^{a} \overline{\mathbf{p}}^{\prime}\right)=\operatorname{LM}(\overline{\mathbf{u}})$ for all $i$ such that $\tau_{i} \neq 0$. So

$\mathbf{L T}(\overline{\mathbf{u}})=\left(\operatorname{LT}(\overline{\mathbf{u}})-\mathbf{L T}\left(k x^{a} \overline{\mathbf{p}}^{\prime}\right)\right)+\operatorname{LT}\left(k x^{a} \overline{\mathbf{p}}^{\prime}\right)=\sum_{i \in J_{\mathrm{LM}(\overline{\mathbf{u}})}}\left(t_{i}+\tau_{i}\right) \operatorname{LT}\left(\overline{\boldsymbol{\alpha}}_{i}\right)$,

and $\mathbf{u}$ is $\mathfrak{s}$-reducible which is a contradiction.

\subsection{Proof of Termination}

The usual proofs of termination of signature-based Gröbner basis algorithms (e.g. [22, Th. 11]) rely on the fact that all elements which are singular $\mathfrak{s}$-reducible are discarded in the computations. Algorithm SigMöller only discards those which are 1-singular $\mathfrak{s}$-reducible. For this reason, we adapt the proof of termination of Algorithm RB [10, Th. 20], which handles singular $\mathfrak{s}$-reducible elements in a different way.

\section{Theorem 5.6 Algorithm SigMöller terminates.}

Proof Let $\mathcal{G}=\left(\boldsymbol{\alpha}_{1}, \ldots, \boldsymbol{\alpha}_{t}, \ldots\right)$ be the sequence of basis elements computed by SigMöller. By construction, for all $t \geq 1, \overline{\boldsymbol{\alpha}_{t}}$ is not $\mathfrak{s}$-reducible by $\mathcal{G}_{t-1}:=\left\{\boldsymbol{\alpha}_{1}, \ldots, \boldsymbol{\alpha}_{t-1}\right\}$, and all $\mathbf{v} \in A^{m}$ with $\mathfrak{s}(\mathbf{v}) \prec \mathfrak{s}\left(\boldsymbol{\alpha}_{t}\right)$ s-reduce to zero w.r.t. $\mathcal{G}_{t-1}$.

For $i \geq 1$, let $M(i)=\operatorname{LM}\left(\overline{\boldsymbol{\alpha}}_{i}\right), T(i)=\mathrm{LT}\left(\overline{\boldsymbol{\alpha}}_{i}\right)$. We define the sig-lead ratio $r\left(\boldsymbol{\alpha}_{i}\right)$ of $\boldsymbol{\alpha}_{i}$ as $\frac{\mathfrak{s}\left(\boldsymbol{\alpha}_{i}\right)}{M(i)}$. Those ratios are ordered naturally by $\frac{s}{m} \prec \frac{s^{\prime}}{m^{\prime}} \Longleftrightarrow s m^{\prime} \prec s^{\prime} m$.

We partition $\mathcal{G}$ into subsets $\mathcal{G}_{r}=\left\{\boldsymbol{\alpha}_{i} \mid r\left(\boldsymbol{\alpha}_{i}\right) \simeq r\right\}$, where $\simeq$ denotes equality up to a coefficient in $R$. We prove that only finitely many $\mathcal{G}_{r}$ are non-empty, and that they are all finite, hence $\mathcal{G}$ is finite.

First, we prove that only finitely many $\mathcal{G}_{r}$ are non-empty. We do so by counting minimal basis elements, where $\boldsymbol{\alpha}_{i}$ is minimal if and only if there is no $\boldsymbol{\alpha}_{j} \in \mathcal{G}$ with $\mathfrak{s}\left(\boldsymbol{\alpha}_{j}\right) \mid \mathfrak{s}\left(\boldsymbol{\alpha}_{i}\right)$ and $T(j) \mid T(i)$. A non-minimal module element $\boldsymbol{\alpha}_{i}$ is $\mathfrak{s}$-reducible by $\left\{\boldsymbol{\alpha}_{1}, \ldots, \boldsymbol{\alpha}_{i-1}\right\}$ [22, Lem. 12], and since all basis elements are regular $\mathfrak{s}$-reduced by construction, $\boldsymbol{\alpha}_{i}$ is singular $\mathfrak{s}$-reducible. In particular, there exists at least one $\boldsymbol{\alpha}_{j}, j<i$ and a monomial $m$ with $\mathfrak{s}\left(m \boldsymbol{\alpha}_{j}\right) \simeq \mathfrak{s}\left(\boldsymbol{\alpha}_{i}\right)$ and $m M(j)=M(i)$, so $\boldsymbol{\alpha}_{i}$ and $\boldsymbol{\alpha}_{j}$ lie in the same subset $\mathcal{G}_{r}$. Hence there are at most as many non-empty $\mathcal{G}_{r}$ 's as there are minimal basis elements. This is finitely many because $A$ and $A^{m}$ are Noetherian.

Then we prove by induction on the finitely many non-empty sets $\mathcal{G}_{r}$ that each $\mathcal{G}_{r}$ is finite. Let $r$ be a sig-lead ratio, assume that for all $r^{\prime}<r, \mathcal{G}_{r^{\prime}}$ is finite. Let $\boldsymbol{\alpha}_{t} \in \mathcal{G}_{r}$. If $\boldsymbol{\alpha}_{t}$ is $\mathbf{e}_{i}$ for some $i$, then it only counts for one. Otherwise, let $J$ be the regular saturated set, and $\mathbf{p}$ the corresponding S-polynomial, that SigMöller regular s-reduced to obtain $\boldsymbol{\alpha}_{t}$. Then $\mathbf{p}=\sum_{j \in J} b_{j} \frac{M(J)}{M(j)} \boldsymbol{\alpha}_{j}$ for $b_{j} \in R$, and there exists $\tau \in J$ such that for all $j \in J \backslash\{\tau\}, \frac{M(J)}{M(j)} \mathfrak{s}\left(\boldsymbol{\alpha}_{j}\right) \prec \frac{M(J)}{M(\tau)} \mathfrak{s}\left(\boldsymbol{\alpha}_{\tau}\right)$. Also $T(t) \prec \operatorname{LT}\left(\frac{M(J)}{M(\tau)} \overline{\boldsymbol{\alpha}}_{\tau}\right)$ and $\mathfrak{s}\left(\boldsymbol{\alpha}_{t}\right)=\frac{M(J)}{M(\tau)} \mathfrak{s}\left(\boldsymbol{\alpha}_{\tau}\right)$. So $r=\frac{\mathfrak{s}\left(\boldsymbol{\alpha}_{t}\right)}{M(t)} \succ \frac{\mathfrak{s}\left(\boldsymbol{\alpha}_{\tau}\right)}{M(\tau)} \succ \frac{\mathfrak{s}\left(\boldsymbol{\alpha}_{j}\right)}{M(j)}$ for $j \in J \backslash\{\tau\}$. Hence all $\boldsymbol{\alpha}_{j}$, $j \in J$ are in some $\mathcal{G}_{r_{j}}$ with $r_{j}<r$, so for computing elements of $\mathcal{G}_{r}$, the algorithm will consider at most as many saturated subsets as there are subsets of $\bigcup_{r^{\prime}<r} \mathcal{G}_{r}$, which is finite by induction. Furthermore, since $R$ is a PID and in particular Noetherian, with each saturated subset $J$, the algorithm only builds finitely many S-polynomials (actually, it only builds one). So overall, we find that $\mathcal{G}_{r}$ is finite, which concludes the proof by induction. 
5.4 Eliminating S-Polynomials

It is well known in the case of fields that additional criteria can be implemented to detect that a regular S-pair will lead to an element which $\mathfrak{s}$-reduces to 0 . In this section, we show how we can implement three such criteria, namely the syzygy criterion, the F5 criterion and the singular criterion.

\subsubsection{Syzygy Criterion}

Syzygy criteria rely on the fact that, if the signature of an S-polynomial can be written as a linear combination of signatures of syzygies, then this S-polynomial would be a syzygy itself. Signatures of syzygies can be identified in two ways:

- the Koszul syzygy between basis elements $\mathbf{p}$ and $\mathbf{q}$ such that $\mathfrak{s}(\mathbf{p})=m_{\mathbf{p}} \mathbf{e}_{i}, \mathfrak{s}(\mathbf{q})=m_{\mathbf{q}} \mathbf{e}_{j}, i<j$ is $\overline{\mathbf{p}} \mathbf{q}-\overline{\mathbf{q}} \mathbf{p}$, and it has signature $\operatorname{LT}(\overline{\mathbf{p}}) \mathfrak{s}(\mathbf{q})$;

- if a regular S-polynomial $\mathbf{p} \mathfrak{s}$-reduces to 0 , then $\mathfrak{s}(\mathbf{p})$ and its multiples are signatures of syzygies; thus, the algorithm may maintain a set of generators of signatures of syzygies by adding to this set $\mathfrak{s}(\mathbf{p})$ for each $\mathrm{S}$ polynomial $\mathbf{p}$ s-reducing to 0 .

For regular sequences, all syzygies are Koszul syzygies.

Proposition 5.7 (Syzygy criterion) Assume that $\mathbf{T}$ is a signature such that all module elements with signature less than $\mathbf{T}$ s-reduce to 0 . Let $\mathbf{p} \in A^{m}$ be such that there exist syzygies $\mathbf{z}_{1}, \ldots, \mathbf{z}_{k}$ and terms $m_{1}, \ldots, m_{k}$ in $A$ with $\mathfrak{s}(\mathbf{p})=\sum_{i=1}^{k} m_{i} \mathfrak{s}\left(\mathbf{z}_{i}\right)$, and $\mathfrak{s}(\mathbf{p}) \preceq \mathbf{T}$. Then $\mathbf{p}$ regular $\mathfrak{s}$-reduces to 0 .

Proof Let $\mathbf{r}=\mathbf{p}-\sum_{i=1}^{k} m_{i} \mathbf{z}_{i}$, then $\mathfrak{s}(\mathbf{r}) \prec \mathfrak{s}(\mathbf{p}) \preceq \mathbf{T}$ so $\mathbf{r} \mathfrak{s}$-reduces to 0 . But $\overline{\mathbf{r}}=\overline{\mathbf{p}}-\sum_{i=1}^{k} m_{i} \overline{\mathbf{z}}_{i}=\overline{\mathbf{p}}$, so $\mathbf{p}$ also $\mathfrak{s}$-reduces to 0 with reducers of signature at most $\mathfrak{s}(\mathbf{r}) \prec \mathfrak{s}(\mathbf{p})$.

Koszul syzygies can be eliminated with the same technique, but it is more efficient to use the F5 criterion [22, Sect. 3.3].

Proposition 5.8 (F5 criterion, [3,11]) Let $\mathbf{p} \in A^{m}$ with signature $\mu \mathbf{e}_{i}$, and let $\left\{\boldsymbol{\alpha}_{1}, \ldots, \boldsymbol{\alpha}_{t}\right\}$ be a signature Gröbner basis of $\left\langle f_{1}, \ldots, f_{i-1}\right\rangle$. Then $\mathbf{p}$ is a Koszul syzygy if and only if $\mu$ is $\mathfrak{s}$-reducible modulo $\left\{\boldsymbol{\alpha}_{1}, \ldots, \boldsymbol{\alpha}_{t}\right\}$.

Proof By definition, $\mathbf{p}$ is a Koszul syzygy if and only if $m \in \mathrm{LT}\left(\left\langle f_{1}, \ldots, f_{i-1}\right\rangle\right)$, and the conclusion follows by definition of a weak Gröbner basis.

\subsubsection{Singular Criterion}

The singular criterion states that the algorithm only needs to consider one S-polynomial with a given signature. So when computing a new S-polynomial, if there already exists a $\mathfrak{s}$-reduced module element with the same signature, we may discard the current S-polynomial without performing any $\mathfrak{s}$-reduction.

Proposition 5.9 (Singular criterion) Let $\mathcal{G}=\left\{\boldsymbol{\alpha}_{1}, \ldots, \boldsymbol{\alpha}_{s}\right\}$ be a signature Gröbner basis up to signature $\mathbf{T}$. Let $\mathbf{p} \in A^{m}$ be such that there exists $\boldsymbol{\alpha}_{i} \in \mathcal{G}$ with $\mathfrak{s}\left(\boldsymbol{\alpha}_{i}\right)=\mathfrak{s}(\mathbf{p})$ and $\mathfrak{s}(\mathbf{p})=\mathfrak{s}(\mathbf{T})$. Then $\mathbf{p} \mathfrak{s}$-reduces to 0 .

Proof Let $\mathbf{p}^{\prime}$ be the result of regular $\mathfrak{s}$-reducing $\mathbf{p}$ w.r.t. $\mathcal{G}$. By construction, the basis element $\boldsymbol{\alpha}_{i}$ is regular $\mathfrak{s}$-reduced w.r.t. $\mathcal{G}$. So by Lemma 5.3, $\operatorname{LM}\left(\overline{\mathbf{p}}^{\prime}\right)=\operatorname{LM}\left(\overline{\boldsymbol{\alpha}}_{i}\right)$, and applying Lemma 4.5, with $k=1$ and $x^{a}=1$, shows that $\mathbf{p}^{\prime}$ is 1 -singular $\mathfrak{s}$-reducible. The result of that $\mathfrak{s}$-reduction has signature $\prec \mathfrak{s}(\mathbf{p})=\mathbf{T}$, so it $\mathfrak{s}$-reduces to 0 . 
Table 1 Computation of a grevlex GB of the Katsura- 2 system in $\mathbb{Z}\left[X_{1}, X_{2}, X_{3}\right]$

\begin{tabular}{lccc}
\hline Algorithm & Pairs/sat. sets & S-polynomials & Reductions to 0 \\
\hline Möller strong & 78 & 20 & 7 \\
SigMöller (with criteria) & 170 & 13 & 0 \\
\hline
\end{tabular}

Table 2 Computation of a grevlex GB of the Katsura-3 system in $\mathbb{Z}\left[X_{1}, X_{2}, X_{3}, X_{4}\right]$

\begin{tabular}{lcrr}
\hline Algorithm & Pairs/sat. sets & S-polynomials & Reductions to 0 \\
\hline Möller strong & 861 & 246 & 159 \\
SigMöller (with criteria) & 2227 & 51 & 0 \\
\hline
\end{tabular}

\section{Experimental Results and Future Work}

We have written a toy implementation of Algorithm SigMöller, ${ }^{2}$ with the F5 and Singular criteria. We provide functions LinDecomp and Satldeal for Euclidean rings, fields and multivariate polynomial rings.

Since our focus is on the feasibility of signature-compatible computations and not their efficiency, we give data about the number of considered S-polynomials, saturated sets and reductions to 0 , when computing Gröbner bases over $\mathbb{Z}$ for the polynomial systems Katsura-2 (Table 1) and Katsura-3 (Table 2). The statistics are compared with a run of Möller's strong algorithm [19]. Even though the proposed algorithm, adapted from Möller's weak algorithm, considers more saturated sets than Möller's strong algorithm, thanks to the signatures, it ends up computing and reducing significantly less S-polynomials, and no reductions to zero appear.

Running Algorithm SigMöller on larger examples would require optimizations, but it appears that the most expensive step is the generation of the saturated sets, which takes time exponential in the size of the current basis. This step may be accelerated in different ways. First, it is known that in the case of PIDs, the reductions of Möller's algorithm can be recovered from those of Möller's strong algorithm [1, Sec. 4.4], which may allow to run the algorithms considering only pairs instead of arbitrary tuples of polynomials. Additionally, Gebauer and Möller's criteria for fields can be used to make Möller's strong algorithm over PIDs more efficient [19]. We will investigate whether it is possible to prove that these algorithms are compatible with signatures in the future. Finally, future research will be focused on further signature-based criteria, such as the cover criterion described in [14] and the more general rewriting criteria.

The algorithm accepts as input polynomials over any ring, provided that the necessary routines are defined. In particular, our implementation can run the algorithms on polynomials on the base ring $\mathbb{K}\left[y_{1}, \ldots, y_{k}\right]$. On small examples in this setting, it appears that the algorithm terminates and returns a correct output. Understanding the behavior of SigMöller over UFDs or even more general rings will also be the focus of future research.

Acknowledgements Open access funding provided by Johannes Kepler University Linz. The authors thank C. Eder and anonymous referees for helpful suggestions, M. Ceria and T. Mora for a fruitful discussion on the syzygy paradigm for Gröbner basis algorithms, and M. Kauers for his valuable insights and comments all through the elaboration of this work.

Open Access This article is distributed under the terms of the Creative Commons Attribution 4.0 International License (http:// creativecommons.org/licenses/by/4.0/), which permits unrestricted use, distribution, and reproduction in any medium, provided you give appropriate credit to the original author(s) and the source, provide a link to the Creative Commons license, and indicate if changes were made.

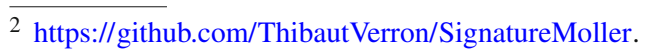




\section{References}

1. Adams, W., Loustaunau, P.: An Introduction to Gröbner Bases, vol. 7. American Mathematical Society, Providence (1994)

2. Arri, A., Perry, J.: The F5 criterion revised. J. Symb. Comput. 46(9), 1017-1029 (2011)

3. Bardet, M., Faugère, J.-C., Salvy, B.: On the complexity of the $F_{5}$ Gröbner basis algorithm. J. Symb. Comput. 70, 49-70 (2015)

4. Bosma, W., Cannon, J., Playoust, C.: The Magma algebra system. I. The user language. J. Symb. Comput. 24(3-4), 235-265 (1997). Computational algebra and number theory (London, 1993)

5. Buchberger, B.: Ein Algorithmus zum Auffinden der Basiselemente des Restklassenringes nach einem nulldimensionalen Polynomideal. PhD thesis, University of Innsbruck, Austria (1965)

6. Eder, C., Faugère, J.-C.: A survey on signature-based algorithms for computing Gröbner bases. J. Symb. Comput. 80, 719-784 (2017)

7. Eder, C., Perry, J.: F5C: a variant of Faugere's F5 algorithm with reduced Gröbner bases. J. Symb. Comput. 45(12), 1442-1458 (2010)

8. Eder, C., Perry, J.: Signature-based algorithms to compute Gröbner bases. In: Proceedings of the 36th International Symposium on Symbolic and Algebraic Computation, pp. 99-106. ACM (2011)

9. Eder, C., Pfister, G., Popescu, A.: On signature-based Gröbner bases over Euclidean rings. In: Proceedings of the 2017 ACM on International Symposium on Symbolic and Algebraic Computation, ISSAC '17, New York, NY, USA, pp. 141-148. ACM (2017)

10. Eder, C., Roune, B.H.: Signature rewriting in Gröbner basis computation. In: Proceedings of the 38th International Symposium on Symbolic and Algebraic Computation, pp. 331-338. ACM (2013)

11. Faugère, J.-C.: A new efficient algorithm for computing Gröbner bases without reduction to zero (F5). In: Proceedings of the 2002 International Symposium on Symbolic and Algebraic Computation, ISSAC '02, New York, NY, USA, pp. 75-83. ACM (2002)

12. Francis, M., Dukkipati, A.: On ideal lattices, Gröbner bases and generalized hash functions. J Algebra Appl (2017)

13. Gao, S., Guan, Y., Volny IV, F.: A new incremental algorithm for computing Gröbner bases. In: Proceedings of the 2010 International Symposium on Symbolic and Algebraic Computation, ISSAC '10. ACM, pp. 13-19 (2010)

14. Gao, S., Volny IV, F., Wang, M.: A new framework for computing Gröbner bases. Math. Comput. 85(297), 449-465 (2016)

15. Gebauer, R., Möller, H.M.: On an installation of Buchberger's algorithm. J. Symb. Comput. 6(2-3), 275-286 (1988)

16. Kandri-Rody, A., Kapur, D.: Computing a Gröbner basis of a polynomial ideal over a Euclidean domain. J. Symb. Comput. 6(1), 37-57 (1988)

17. Lichtblau, D.: Effective computation of strong Gröbner bases over Euclidean domains. Ill. J. Math. 56(1), 177-194 (2013). 2012

18. Lichtblau, D.: Applications of strong Gröbner bases over Euclidean domains. Int. J. Algebra 7(5-8), 369-390 (2013)

19. Möller, H.M.: On the construction of Gröbner bases using syzygies. J. Symb. Comput. 6(2-3), 345-359 (1988)

20. Möller, H.M., Mora, T., Traverso, C.: Gröbner bases computation using syzygies. In: Papers from the International Symposium on Symbolic and Algebraic Computation, ISSAC '92, New York, NY, USA, pp. 320-328. ACM (1992)

21. Nabeshima, K.: Reduced Gröbner bases in polynomial rings over a polynomial ring. Math. Comput. Sci. 2(4), 587-599 (2009)

22. Roune, B.H., Stillman, M.: Practical Gröbner basis computation. In: Proceedings of the 37th International Symposium on Symbolic and Algebraic Computation, pp. 203-210. ACM (2012)

23. Zacharias, G.: Generalized Gröbner bases in commutative polynomial rings. Master's thesis, MIT, Cambridge, MA (1978)

Publisher's Note Springer Nature remains neutral with regard to jurisdictional claims in published maps and institutional affiliations. 